\title{
Programa de intervención en debate crítico sobre el pensamiento crítico en universitarios
}

\section{Intervention program based on critical debate on the critical thinking of university} students

Sonia Betancourth Zambrano

Yuranny Tabares Díaz ii

Viviana Martínez Daza i

Universidad de Nariño, Pasto, Colombia

\section{Resumen}

Objetivo: analizar los efectos de un programa de intervención basado en debate crítico sobre el pensamiento crítico de estudiantes universitarios. Método: estudio cuasi experimental con mediciones pre y post con único grupo. Se trabajó con estudiantes de psicología, a quienes se aplicó la prueba de pensamiento crítico PENCRISAL. El programa de intervención se realizó en seis sesiones de trabajo, brindando además asesorías presenciales y a través de medios informáticos y redes sociales. Resultados: se evidenció una mejora significativa en el pensamiento crítico de los universitarios posterior al programa implementado, en los factores toma de decisiones, solución de problemas y puntuación total de la prueba. Discusión y Conclusiones: el debate crítico privilegia el desarrollo de este tipo de pensamiento y sus habilidades en la población estudiantil.

Palabras clave: debate crítico, estudiantes universitarios, pensamiento crítico.

\section{Abstract}

Objective: this work aims to analyse the effects of an intervention program based on critical debate on the critical thinking of university students. Method: quasiexperimental study with pre and post group measurements. This one was worked with students of psychology, to whom the test of critical thought PENCRISAL was applied. The intervention program was carried out in six work sessions, in which advice and information were also offered through computer media and social networks. Results: There was a significant improvement in the critical thinking of university students after the implementation of the program, in the factors of decision making, problem solving and total test score. Discussion y Conclusion: critical debate privileges the development of this type of thinking and its capacities in the student population.

Keywords; critical debate, university students, critical thinking.
Open Access:

ISSN: $0124-212$ E-ISSN: $2665-2420$

ARTÍCULO RSULTADO DE INVESTIGACIÓN
Copyright @ 2020 By Educación y Humanismo

Editor: Patricia Martínez Barrios Universidad Simón Bolívar

\section{Correspondencia: \\ Yuranny Tabares alejataba15@hotmail.com}

Recibido: 28-08-2019 Aceptado: $28-10-2019$ En línea desde: 02-12-2019

DOI:

10.17081/eduhum. 22 38.3577

Cómo citar este artículo (APA): Betancourth, S., Tabares, Y. \& Martínez, V. (2020). Programa de intervención en debate crítico sobre el pensamiento crítico en universitarios. Educación y Humanismo, 22(38), 1-17. DOI: 


\section{Introducción}

El pensamiento crítico es definido por Saiz \& Rivas (2008) como un proceso orientado a la búsqueda de conocimiento, por medio de habilidades de razonamiento, toma de decisiones y solución de problemas, con el objetivo de alcanzar con eficacia los resultados deseados. En este sentido, este tipo de pensamiento requiere el cumplimiento de estándares de calidad y control en cuanto a interpretación, inferencia, evaluación, análisis y reflexión, propendiendo por el planteamiento de posiciones razonables y justificadas frente a las situaciones o tópicos en cuestión (Paul \& Elder, 2003; Creamer, 2011; Rivas \& Saiz, 2016).

A nivel latinoamericano, investigaciones como la de Betancourth, Muñoz y Rosas (2017) y Ossa, Palma, Lagos y Díaz (2018) en estudiantes universitarios de instituciones de educación pública en Chile, arrojan como principales resultados la presencia de bajos niveles de pensamiento crítico en los participantes, particularmente en habilidades fundamentales para el logro del razonamiento científico, tales como, la indagación, el análisis, la argumentación y la toma de decisiones.

De manera similar, ejercicios investigativos con estudiantes universitarios de Perú de los programas de ingeniería económica, estadística y ciencias sociales, dan cuenta de la existencia de niveles medios y bajos de pensamiento crítico, preponderantemente en la dimensión de inferencia (Dávila, 2018). Los hallazgos señalados, son equiparables a los encontrados por Lozano y Cerrón (2015), los cuales permiten concluir que el $53.97 \%$ de la muestra de estudiantes de la facultad de educación se ubicaron en un nivel medio de pensamiento crítico.

Por su parte, Bejarano, Galván y López (2013) en su investigación sobre destrezas intelectuales y motivaciones hacia las mismas, en estudiantes de psicología de Barrancabermeja-Colombia, reporta que el nivel de competencias en pensamiento crítico es bajo, tanto en el desempeño total como en sus dimensiones, denotando con ello un manejo deficiente de estas por parte de los evaluados.

Desde esta perspectiva, los estudios descritos anteriormente permiten concluir que en el contexto latinoamericano de la educación superior, predomina la presencia de niveles bajos y medios de pensamiento crítico, corroborando a su vez que el proceso de aprendizaje de los universitarios no promueve la adquisición y fortalecimiento de habilidades intelectuales, indispensables para el afrontamiento de las situaciones cotidianas y el posicionamiento crítico y reflexivo, necesario para dar solución a las problemáticas en las que se hallan inmersos (Lander, 2001; Bejarano et al., 2013; Minte \& Ibagón, 2017; Steffens, Ojeda, Martínez, Hernández \& Moronta, 2018).

En contraste, las pruebas de ingreso a las Instituciones de Educación Superior (IES), evidencian en sus resultados que si bien el rendimiento de los universitarios colombianos se ubica por igual o encima de la media a nivel nacional, el grado de desarrollo de pensamiento 
crítico es bajo, factor que dista de la cantidad de años de formación escolarizada recibida desde preescolar hasta pregrado y de las competencias y habilidades que se esperaría deberían desarrollarse (Melo, Ramos \& Hernández, 2017; Steffens et al., 2018). A ello se suma, que el perfil de ingreso de los estudiantes a las IES no es afín al esperado, dado que se vislumbran dificultades en cuanto a lectura comprensiva y resolución de problemas de lógica-matemática (Consejo Privado de Competitividad, 2013; Castro, Ruíz \& Guzmán, 2018).

De este modo, la expectativa se dirige a que las IES asuman el rol de educar no sólo en la disciplina seleccionada por los estudiantes, sino también en el desarrollo de niveles adecuados de competencias cognitivas e intelectuales, puesto que es notorio que gran parte del sistema educativo ha dado continuidad a prácticas tradicionales y memorísticas, caracterizadas por instaurar aprendizajes fragmentados, acríticos y desactualizados que imposibilitan la articulación de los conceptos, desmotivando y obstaculizando conjuntamente la curiosidad de los estudiantes y el fortalecimiento de sus habilidades (Acosta, 2002; Vélez, 2013; Santiago, 2016; Aznar \& Laiton, 2017).

En este orden de ideas, el contexto de globalización y sus exigencias, hacen manifiesta la importancia del pensamiento crítico y su vinculación a los procesos educativos, en aras de que estudiantes y docentes trabajen de forma conjunta en mecanismos promotores de la comprensión y aplicación de los conocimientos en diferentes escenarios, posibilitando la reorganización y reestructuración de la información y, por ende, la asignación de sentido a lo que se piensa, siente y hace (Paul \& Elder, 2008; López, 2012; Lara \& Rodríguez, 2016; Núñez, Ávila \& Olivares, 2017).

Así también, la educación superior actual ha reconocido la necesidad de proporcionar a los estudiantes una formación integral basada en competencias transversales, aplicadas a todos los niveles educativos, las cuales sean atingentes a las demandas del entorno y aporten a la consolidación de futuros profesionales al servicio de la sociedad (Prieto, 2008; Beltrán \& Torres, 2009; Monarca, 2013; Olivares, 2016). A partir de ello, se ha instituido la responsabilidad a los centros educativos de proveer herramientas útiles a los estudiantes para que aprendan a pensar mejor e igualmente, de manera efectiva y eficiente (Hawes, 2003; Amador, 2012; Willson, 2012).

Dentro de dichas herramientas, se destaca el desarrollo del pensamiento crítico a través de estrategias de enseñanza, las cuales hacen alusión a actos que el docente ejecuta para fomentar la consecución de aprendizajes significativos en los estudiantes y consecuentemente, promover su autonomía (Guzmán \& Sánchez, 2008; Buitrago, Higuita \& Moreno, 2010; Palacios, Álvarez, Moreira \& Morán, 2017).

En este marco, Montoya y Monsalve (2008) en su investigación en estudiantes de educación secundaria de modalidad virtual de una universidad colombiana, orientado a reconocer herramientas para el fomento del pensamiento crítico, concluye que una labor esencial en la docencia corresponde a la generación de espacios de aprendizaje para el modelamiento del 
espíritu crítico, independientemente del área o el nivel académico.

A la par, Betancourth, Enríquez y Castillo (2010) en su estudio tendiente a analizar los efectos de un programa de intervención basado en la controversia-socrática, sobre el pensamiento crítico en universitarios de psicología del suroccidente colombiano, afirma que a diferencia de las metodologías tradicionales y conductistas usualmente implementadas en el aula de clases, dicha estrategia permite dinamizar las habilidades intelectuales, propiciando además las condiciones idóneas para su desarrollo,

De esta forma, las investigaciones presentadas hacen explicita la viabilidad de crear propuestas para el fortalecimiento de este proceso cognitivo, realizando una evaluación de los efectos que trae consigo la implementación de estrategias de pensamiento crítico y su incidencia en el mejoramiento de las prácticas curriculares (Laiton, 2010; González, 2013; Roca, 2013; Moreno y Velázquez, 2017). Dentro de estas estrategias, se destaca el debate crítico, puesto que permite desarrollar habilidades de argumentación, contra argumentación, respeto por las diferentes posiciones, pensamiento rápido, entre otras (Freeley \& Steinberg, 2009; Fuentes, 2011).

En consonancia con lo anterior, resulta relevante investigar en profundidad sobre esta estrategia y sus efectos en el pensamiento crítico, con el propósito de ampliar y realizar aportes a nivel conceptual y disciplinar, más aún en el contexto colombiano donde se entrevé la existencia de pocos estudios en relación a procesos de intervención o entrenamiento en el mismo (Steffens et al., 2018). Por tal razón, la presente investigación tuvo como objetivo analizar los efectos del debate crítico sobre el pensamiento crítico de estudiantes universitarios.

\section{Método}

\section{Tipo de estudio y diseño}

Estudio cuantitativo de corte cuasi experimental con pre y post-prueba con un solo grupo (O1-X-O2) (Bono, 2012; Manterola \& Otzen, 2015).

\section{Variables de estudio}

La variable dependiente del estudio fue el pensamiento crítico, compuesto por cinco factores o habilidades: Razonamiento Deductivo (RD), Razonamiento Inductivo (RI), Razonamiento Práctico (RP), Toma de Decisiones (TD) y Solución de Problemas (SP). Por su parte, la variable independiente correspondió al programa de intervención basado en el debate crítico.

\section{Participantes}

Se trabajó con 20 estudiantes de psicología de séptimo semestre (A-2019) de la Universidad de Nariño-Colombia, inscritos en el curso de psicología educativa II. No obstante, la muestra 
estadísticamente aceptada se concretó en 18 estudiantes, que participaron de todo el programa y respondieron el test en su medición pre y post. El estudio utilizó una muestra de conveniencia basada en la accesibilidad (Hernández, Fernández \& Baptista, 2006; Creswell, 2014).

\section{Instrumento de recolección de información}

Se empleó la versión adaptada a Perú de la prueba de pensamiento crítico PENCRISAL (Rivas, Morales \& Saiz, 2014), la cual fue creada originalmente en España (Rivas \& Saiz, 2012). Ésta se compone de 35 reactivos que exponen problemáticas de la cotidianidad en diversos temas; las preguntas están organizadas en 5 factores que aluden a las habilidades de pensamiento crítico, con 7 ítems cada uno, presentados en forma aleatoria, estos son: Razonamiento Deductivo(RD), Razonamiento Inductivo(RI), Razonamiento Práctico(RP), Toma de Decisiones(TD) y Solución de Problemas(SP).

El formato de respuesta a las preguntas es abierto, siendo necesario justificar cada una de las mismas. La corrección y puntuación utiliza criterios estandarizados, fijando valores así: 0 puntos (la solución presentada a la problemática es incorrecta); 1 punto (la solución expuesta es correcta, pero carece de argumentación suficiente); 2 puntos (la solución planteada es correcta y su argumentación es adecuada). El sistema de escalamiento es cuantitativo con valores de 0-70 puntos para el puntaje total del test y de 0-14 para sus factores (Rivas \& Saiz, 2012).

EI PENCRISAL es una prueba de potencia, con un tiempo estimado de resolución total de 60 a 90 minutos. La forma de administración más habitual es la informatizada, por internet, con aplicación individual, aunque puede realizarse también en formato papel y lápiz de manera colectiva (Rivas \& Saiz, 2012).

En relación a las propiedades psicométricas de la prueba en su adaptación peruana, la consistencia interna estimada mediante alfa de Cronbach fue de .734, altamente significativa con un p-valor <.001; la fiabilidad interjueces arrojó una concordancia elevada con un coeficiente Kappa de Cohen en un rango de .511 a .970 ( $p$-valor <.001); el análisis factorial evidenció adecuado ajuste al modelo teórico de partida, acorde a la estructura del test original; finalmente, la correlación con otros instrumentos apoyó la validez divergente de la prueba (Rivas et al., 2014).

\section{Procedimiento}

\section{Descripción del modelo de debate crítico.}

El programa de intervención empleó la estructura de debate crítico propuesta por la Universidad Diego Portales-Chile (Fuentes, 2011), modelo centrado en una argumentación desde el sentido pragma-dialéctico (Freeley \& Steinberg, 2009; Ramos, 2019). De este modo, supone la conformación de tres bancadas: investigadora, a favor y en contra, siguiendo 
consecutivamente cuatro fases: reporte de investigación, debate cerrado, debate abierto y cierre (Fuentes, 2011; Arcila et al., 2017).

Cabe mencionar que se realizaron adaptaciones al modelo, en tiempos y orden de intervención de las bancadas. A su vez, se conformaron dos equipos críticos, integrados por las tres bancadas respectivas, cada uno de los cuales participó de tres debates críticos, conservando sus integrantes en el desarrollo de los mismos, pero con roles rotativos.

\section{Descripción del programa de intervención en debate crítico.}

Se solicitó autorización por escrito a los participantes a través de un consentimiento informado. A partir de ello, se realizó la aplicación del pre-test vía web de la prueba PENCRISAL, entregando la dirección electrónica, clave de acceso e indicaciones para el diligenciamiento. Posteriormente, se desarrolló el programa de intervención organizado en cuatro actividades de trabajo, con una extensión de seis sesiones; finalmente, una semana después del programa de intervención, se aplicó el post-test, con las mismas indicaciones previstas en el pre-test.

La primera actividad que integró el programa de intervención correspondió a la realización de asesorías presenciales, las cuales consistieron en un encuentro semanal previo a los debates, para el fortalecimiento de habilidades como argumentación, contra-argumentación e identificación y planteamiento de posibles soluciones a situaciones problemáticas (Mota, 2010; Albornoz, 2017). En la segunda actividad se realizaron asesorías mediante medios informáticos y redes sociales, para lo cual se dispuso de un espacio virtual (correo electrónico, redes sociales y un curso virtual en Moodle) (Godoy \& Calero, 2018), usado para la entrega de material de apoyo, resolución de dudas, recepción de retroalimentaciones de los informes investigativos, entre otros.

La tercera actividad incluyó cinco sesiones: en la primera, se efectuó la introducción del programa de entrenamiento y metodología y en las cuatro sesiones subsiguientes, se desarrollaron los debates críticos (debate crítico 1 y 2, simulacro gran debate crítico y gran debate crítico), enmarcados en las siguientes temáticas, con dos premisas para cada uno de estos: educación (historia, objetivos, metas, conflictos), papel de la psicología en la educación y educación en Colombia respectivamente, siguiendo metodológicamente las etapas del modelo.

El proceso fue evaluado a través de rubricas y pautas de cotejo, elaboradas por el equipo investigador, así como a través del feedback brindado a los equipos (Drago, 2017). Cabe mencionar, que el gran debate crítico se realizó en un evento público y su evaluación estuvo a cargo de tres jurados capacitados en el modelo, dos de ellos externos al equipo investigador. Por último, la actividad cuatro hizo referencia a la sesión final de trabajo, en la que se evaluó el programa de intervención desde la perspectiva de los estudiantes, reconociendo aspectos positivos y de mejora del mismo, así como aprendizajes y emociones suscitadas. 


\section{Análisis de datos}

Para comprobar la normalidad de los datos se empleó la prueba de Shapiro-Wilk para variables cuantitativas en muestras inferiores a 50 casos y la prueba no paramétrica de rangos con signo de Wilcoxon, la cual permite la verificación de cambios estadísticamente significativos en el pre y pos-test (variables cuantitativas) (Flores, Miranda \& Villasís, 2017).

Por su parte, se aplicaron las pruebas no paramétricas $U$ de Mann-Whitney (variable independiente dicotómica y variable dependiente cuantitativa) y $\mathrm{H}$ de Kruskal Wallis (variable independiente policotómica y variable dependiente cuantitativa) para comparar los resultados del test y su variación en función de las características sociodemográficas (Flores et al., 2017). Cabe mencionar, que se hizo uso de pruebas no paramétricas a pesar de que los datos presentaron una distribución normal, puesto que se trabajó con una muestra pequeña $(n<30$ casos) (San Martín, 2011). La conversión de puntajes brutos a centiles, se llevó a cabo usando los baremos de la adaptación del PENCRISAL a población peruana, dada la similitud a nivel cultural en contraste a otros baremos existentes (Rivas et al., 2014).

\section{Resultados}

\section{Caracterización de los participantes}

La muestra estuvo conformada por 18 universitarios de psicología, de los cuales 66.7\% $(n=12)$ eran mujeres y $33.3 \%(n=6)$ eran hombres. Su edad estaba entre los 19 a 31 años.

Por otra parte, $83.3 \%(n=15)$ de los participantes provenía de instituciones educativas de carácter público y $16.7 \%(n=3)$ de instituciones educativas privadas. Respecto al nivel socioeconómico, $55.6 \%(n=10)$ pertenecía al nivel $1,22.2 \%(n=4)$ al nivel 2 y en un porcentaje equivalente a éste último al nivel 3; dichos niveles corresponden en Colombia a estratos bajo y medio, albergando a las personas con menores recursos.

\section{Análisis descriptivo del PENCRISAL}

Se llevó a cabo un análisis descriptivo de los resultados derivados de las puntuaciones del pre y post-test del PENCRISAL (Tabla 1), cuyos resultados permitieron reconocer que los factores referidos a RP, TD y SP obtuvieron las medias más altas en ambas mediciones.

Tabla 1. Resumen descriptivo del PENCRISAL

\begin{tabular}{|c|c|c|c|c|c|c|}
\hline \multirow[b]{2}{*}{ PENCRISAL } & \multicolumn{3}{|c|}{ Medida pre-test } & \multicolumn{3}{|c|}{ Medida post-test } \\
\hline & Media & $\begin{array}{l}\text { Desviación } \\
\text { estándar }\end{array}$ & $\begin{array}{c}\text { Rango } \\
\text { (Min-Máx) }\end{array}$ & Media & $\begin{array}{l}\text { Desviación } \\
\text { estándar }\end{array}$ & $\begin{array}{c}\text { Rango } \\
\text { (Min-Máx) }\end{array}$ \\
\hline
\end{tabular}




\begin{tabular}{lcccccc}
\hline RD & 3.72 & 1.965 & $0-7$ & 4.39 & 2.279 & $1-8$ \\
RI & 4.67 & 1.749 & $1-8$ & 4.94 & 1.626 & $2-8$ \\
RP & $\mathbf{5 . 7 2}$ & 2.761 & $0-10$ & 6.89 & 3.160 & $0-11$ \\
TD & $\mathbf{5 . 5 0}$ & 1.886 & $2-8$ & 7.17 & 2.203 & $3-11$ \\
SP & $\mathbf{6 . 0 6}$ & 2.555 & $1-10$ & 7.06 & 2.555 & $2-11$ \\
PT & 25.67 & 7.799 & $10-38$ & 30.44 & 8.820 & $13-44$ \\
\hline
\end{tabular}

Nota. $\mathrm{RD}=$ razonamiento deductivo; $\mathrm{RI}=$ razonamiento inductivo; $\mathrm{RP}=$ razonamiento práctico; $\mathrm{TD}=$ toma de decisiones; SP=solución de problemas; PT=puntuación total. Fuente: elaborado por las autoras (2019).

Al realizar una comparación de las medias de la muestra con las del baremo peruano del PENCRISAL, se identificó en el pre-test que los resultados en la puntuación total de la prueba y en sus cinco factores, se ubicaron en los centiles 55 a 65 y en el caso del post-test en los centiles 65 a 80. Específicamente, en el pre-test se obtuvieron los rendimientos más altos en los factores RI, RD, RP y TD con el mismo centil y el más bajo en la puntuación total; y frente al post-test, el factor TD obtuvo el centil más alto y los factores RD y RI los más bajos (Tabla 2).

Tabla 2. Comparación centiles baremo población peruana y estudiantes de psicología

\begin{tabular}{|c|c|c|c|c|c|c|}
\hline & RD & RI & $\mathbf{R P}$ & TD & SP & PT \\
\hline \multicolumn{7}{|c|}{ Baremo para población general (muestra peruana) } \\
\hline $\begin{array}{c}\text { Media } \\
\text { (centil 50) }\end{array}$ & 3.66 & 4.75 & 5.32 & 5.41 & 5.66 & 24.80 \\
\hline \multicolumn{7}{|c|}{ Muestra analizada pre-test $(\mathrm{N}=18)$} \\
\hline Media & 3.72 & 4.67 & 5.72 & 5.50 & 6.06 & 25.67 \\
\hline Centil & 65 & 65 & 65 & 65 & 60 & 55 \\
\hline \multicolumn{7}{|c|}{ Muestra analizada post-test $(\mathrm{N}=18)$} \\
\hline Media & 4.39 & 4.94 & 6.89 & 7.17 & 7.06 & 30.44 \\
\hline Centil & 65 & 65 & 75 & 80 & 75 & 75 \\
\hline
\end{tabular}

Fuente: elaborado por las autoras (2019).

De esta manera, los datos presentados con anterioridad mostraron que el desempeño en pensamiento crítico en sus mediciones pre y post, se encuentran por encima de la media del baremo de la población peruana, tanto en los factores de la prueba como en la puntuación total de la misma.

\section{Efectos del programa de intervención sobre el pensamiento crítico}

Para analizar los efectos obtenidos sobre el pensamiento crítico, se realizó la prueba de contraste de normalidad de Shapiro-Wilk para muestras inferiores a 50 casos, la cual arrojó un p-valor >.05 indicando que el conjunto de datos se distribuía normal (Tabla 3).

Tabla 3. Prueba de contraste de normalidad de Shapiro-Wilk

\begin{tabular}{ccccccc}
\hline & RD & RI & RP & TD & SP & PT \\
\hline $\begin{array}{c}\text { Medida pre-test } \\
\text { Z s-w }\end{array}$ & .959 & .953 & .952 & .928 & .946 & .927 \\
p-sig. & .585 & .474 & .462 & .179 & .360 & .171 \\
\hline $\begin{array}{c}\text { Medida post- } \\
\text { test }\end{array}$ & .919 & .965 & .925 & .928 & .946 & .965
\end{tabular}


Fuente: elaborado por las autoras (2019).

En coherencia a ello, al comparar las medias obtenidas en el puntaje total de la prueba y en los factores que la componen, se evidencia un incremento en dicha medida de tendencia central en el post-test de todas las variables. Por su parte, para identificar la existencia de diferencias significativas en las mediciones, se aplicó la prueba no paramétrica de rangos con signo de Wilcoxon, cuyos datos evidenciaron que la puntuación total de la prueba y los factores TD y SP, mejoraron significativamente después del programa de intervención con un p-valor $<.05$ (Tabla 4).

Tabla 4. Prueba de rangos con signo de Wilcoxon

\begin{tabular}{lcc}
\hline & $\mathbf{Z}$ & $\begin{array}{c}\text { Sig. } \\
\text { asintótica } \\
\text { (bilateral) }\end{array}$ \\
\hline RD post/pre & $-1,196^{\mathrm{b}}$ & .232 \\
RI post/pre &,$- 714^{\mathrm{b}}$ & .475 \\
RP post/pre & $-1,689^{\mathrm{b}}$ & .091 \\
TD post/pre & $-2,861^{\mathrm{b}}$ & $\mathbf{. 0 0 4}$ \\
SP post/pre & $-2,069^{\mathrm{b}}$ & $\mathbf{. 0 3 9}$ \\
PT post/pre & $-2,916^{\mathrm{b}}$ & $\mathbf{. 0 0 4}$ \\
\hline b Se basa en rangos negativos (post $>$ pre) \\
\multicolumn{2}{l}{ Fuente: elaborado por las autoras (2019). }
\end{tabular}

\section{Comparación de resultados del PENCRISAL en función de variables sociodemográficas}

Con el fin de comprobar la existencia de diferencias en el pensamiento crítico de los estudiantes antes y después del programa de intervención, se realizó la prueba no paramétrica $\mathrm{U}$ de Mann-Whitney, para las variables sexo, edad e institución educativa de procedencia de los participantes y la prueba no paramétrica $\mathrm{H}$ de Kruskal Wallis para el nivel socioeconómico.

De este modo, respecto al sexo, edad y procedencia de la institución educativa de los estudiantes, los resultados de la prueba $U$ de Mann-Whitney evidenciaron la no existencia de diferencias entre el género femenino y masculino, entre grupos etarios y entre procedencia de instituciones educativas de carácter público y privado, que fuesen catalogadas como significativas, tanto en los cinco factores como en la puntuación total de la prueba, puesto que el p-valor fue $>.05$ en la medición pre y post, es decir, que no se presentan variaciones en el pensamiento crítico de los universitarios en función de dichas variables sociodemográficas.

Por su parte, la prueba $\mathrm{H}$ de Kruskal Wallis señaló diferenciación significativa a nivel estadístico entre el nivel socioeconómico y la habilidad de RI únicamente en la medición post, arrojando un p-valor <.05 (Tabla 5). En este sentido, se aplicó la prueba post hoc para determinar qué medias difieren entre sí, denotando que en el post-test las diferencias significativas ocurren entre los niveles socioeconómicos 1 y 3 , siendo mayor el rendimiento en 
el factor RI en el nivel socioeconómico más alto.

Tabla 5. Prueba H de Kruskal Wallis (nivel socioeconómico)

\begin{tabular}{|c|c|c|c|c|c|c|c|c|c|}
\hline \multirow[b]{2}{*}{ Var. } & \multirow[b]{2}{*}{$\begin{array}{c}\text { Nivel } \\
\text { Soc-eco }\end{array}$} & \multicolumn{4}{|c|}{ Pre-test } & \multicolumn{4}{|c|}{ Post-test } \\
\hline & & Media & DS & Estadístico & $\begin{array}{c}\text { Sig. } \\
\text { asintótica }\end{array}$ & Media & DS & Estadístico & $\begin{array}{c}\text { Sig. } \\
\text { asintótica }\end{array}$ \\
\hline $\mathrm{RD}$ & $\begin{array}{l}\text { Nivel } 1 \\
\text { Nivel } 2 \\
\text { Nivel } 3\end{array}$ & $\begin{array}{l}3.70 \\
4.00 \\
3.50\end{array}$ & $\begin{array}{l}1.829 \\
2.449 \\
2.380\end{array}$ & .399 & .819 & $\begin{array}{l}3.80 \\
4.75 \\
5.50\end{array}$ & $\begin{array}{l}2.044 \\
2.872 \\
2.380\end{array}$ & 2.198 & .333 \\
\hline RI & Nivel 1 & 4.30 & 1.059 & & & 4.30 & 1.337 & & \\
\hline & $\begin{array}{l}\text { Nivel } 2 \\
\text { Nivel } 3\end{array}$ & $\begin{array}{l}3.75 \\
6.50\end{array}$ & $\begin{array}{l}2.500 \\
1.291\end{array}$ & 5.902 & .052 & $\begin{array}{l}4.75 \\
6.75\end{array}$ & $\begin{array}{c}1.708 \\
.957\end{array}$ & 6.595 & .037 \\
\hline $\mathrm{RP}$ & Nivel 1 & 5.80 & 3.393 & & & 6.60 & 2.989 & & \\
\hline & $\begin{array}{l}\text { Nivel } 2 \\
\text { Nivel } 3 \\
\end{array}$ & $\begin{array}{l}5.25 \\
6.00 \\
\end{array}$ & $\begin{array}{l}2.217 \\
1.826 \\
\end{array}$ & .362 & .835 & $\begin{array}{l}7.00 \\
7.50 \\
\end{array}$ & $\begin{array}{l}3.559 \\
4.041 \\
\end{array}$ & .676 & .713 \\
\hline TD & Nivel 1 & 5.10 & 1.912 & & & 6.60 & 2.271 & & \\
\hline & $\begin{array}{l}\text { Nivel } 2 \\
\text { Nivel } 3\end{array}$ & $\begin{array}{l}6.00 \\
6.00\end{array}$ & $\begin{array}{l}1.414 \\
2.449\end{array}$ & 1.083 & .582 & $\begin{array}{l}8.00 \\
7.75\end{array}$ & $\begin{array}{c}2.944 \\
.957\end{array}$ & 1.038 & .595 \\
\hline SP & Nivel 1 & 5.60 & 2.716 & & & 6.70 & 2.869 & & \\
\hline & Nivel 2 & 7.25 & 2.500 & .837 & .658 & 7.50 & 2.380 & .518 & .772 \\
\hline & Nivel 3 & 6.00 & 2.449 & & & 7.50 & 2.380 & & \\
\hline PT & Nivel 1 & 24.50 & 8.073 & & & 28.00 & 7.614 & & \\
\hline & $\begin{array}{l}\text { Nivel } 2 \\
\text { Nivel } 3\end{array}$ & $\begin{array}{l}26.25 \\
28.00\end{array}$ & $\begin{array}{l}8.995 \\
7.483\end{array}$ & .425 & .808 & $\begin{array}{l}32.00 \\
35.00\end{array}$ & $\begin{array}{c}12.028 \\
8.287\end{array}$ & 1.992 & .369 \\
\hline
\end{tabular}

Fuente: elaborado por las autoras (2019).

\section{Discusión y Conclusión}

La investigación buscó analizar los efectos de un programa de intervención basado en el debate crítico sobre el pensamiento crítico de estudiantes universitarios de psicología. Se aplicó la prueba PENCRISAL que evalúa cinco habilidades de pensamiento crítico.

A nivel descriptivo, se halla que los niveles promedio de la presente muestra tanto en el puntaje total como en los factores que componen la prueba fueron superiores a los de la población normativa peruana, obteniendo centiles superiores a los de ésta (centil 50). Dichos hallazgos se contraponen a los estudios de Anganoy, Pantoja, Jurado, Vallejo y Botina (2017) y Aznar y Laiton (2017) en Colombia, y Robles, Cisneros y Guzmán (2016) en México, en los cuales las puntuaciones de los estudiantes evaluados se ubicaron muy por debajo del centil alcanzado en el baremo del grupo peruano.

En cuanto a los efectos del programa de intervención, se observa que éste permitió incrementar y mejorar significativamente el pensamiento crítico de los estudiantes, específicamente en habilidades como la toma de decisiones, solución de problemas y puntuación total, pero no estadísticamente significativa en razonamiento deductivo, inductivo y práctico. 
Es así como se vislumbra que el debate crítico privilegia el pensamiento crítico en los universitarios, puesto que dentro de los aspectos fuertes de la estrategia se destaca la indagación de información y el pensar rápidamente en búsqueda de mejores argumentos y contraargumentos (Fuentes, 2011; Fuentes \& Santibáñez, 2011). A ello se suma, la consideración de que el debate crítico aporta en la construcción de argumentos positivos y negativos frente a una idea polémica, que involucra al individuo en un proceso que lo conduce a reestructurar su pensamiento (Leitão, De chiaro \& Cano, 2016; Pinto, 2017).

Así también, se evidencia que los resultados del PENCRISAL antes y después del programa de intervención no difieren en función del sexo, edad y procedencia de la institución educativa, aspecto que reafirma que el debate crítico permitió el desarrollo de este pensamiento. Respecto al sexo, hallazgos de Ennis, Millman y Tomko (1985), Bejarano et al. (2013), Anganoy et al. (2017), Loaiza \& Osorio (2018), indican que tanto hombres como mujeres desarrollan el pensamiento crítico por igual, conclusión atingente a la encontrada en el presente estudio.

Por su parte, los hallazgos encontrados con la variable edad son coherentes a los de estudios tales como los de Aznar y Laiton (2017) y Betancourth et al. (2017), los cuales exponen que no existen variaciones en el rendimiento del pensamiento crítico y sus habilidades en función del grupo etario. En contraste, la investigación de Bejarano et al. (2013) asevera que en el factor toma de decisiones del PENCRISAL, los estudiantes menores de 19 años tienen mejor desempeño en dicha habilidad que aquellos con la misma edad o una superior.

En cuanto a la variable procedencia de la institución educativa, se encuentran resultados disimiles a los del presente estudio, dado que investigaciones como las de García y Vázquez (2018) y Loaiza \& Osorio (2018) exhiben que los estudiantes provenientes de instituciones particulares de educación consiguieron mejores resultados en pensamiento crítico que aquellos de instituciones educativas públicas. No obstante, Molina, Morales y Valenzuela (2016) y Vásquez, Pleguezuelos y Mora (2017) prevén la relevancia de que éste se fomente transversalmente, independiente de variables sociodemográficas con las que se halla en interacción, en aras de contrarrestar el afrontamiento pasivo de los estudiantes en su proceso de aprendizaje.

Por otro lado, es importante resaltar la forma cómo se dinamizó el debate crítico, en primer lugar, antes de llevar a cabo los debates se brindó asesoría a cada una de las bancadas; en segundo lugar, uno de los debates se realizó abierto al público, aspecto que motivó a los estudiantes a ejercer un mayor esfuerzo en su trabajo previo al debate y tener una buena preparación para el mismo; y en tercer lugar, la elección de las temáticas, las cuales fueron actualizadas y de gran interés para los estudiantes: la educación, el papel de la psicología en la educación y la educación en Colombia.

Al respecto, Betancourth (2015) manifiesta que las formas de dinamizar las estrategias, se relacionan con la estructura y con el contexto; en este marco, la presente investigación trabajó en la dinamización del contexto con la asesoría, el debate público y las temáticas, puesto que 
el desarrollo de estas habilidades cognitivas e intelectuales no puede desligarse de la estrategia y los contenidos temáticos de las asignaturas.

En coherencia a lo planteado, se concluye que la estrategia de debate crítico puede ser utilizada en las clases universitarias y, por tanto, el pensamiento crítico es susceptible de ser desarrollado en cada asignatura que el estudiante curse. Es así como los estudiantes que reflexionan sobre las temáticas impartidas, lograrán mejores calificaciones, al tomar conciencia de los recursos de los que disponen y emplear los que les resulten más eficientes (Hawes, 2003; Difabio de Anglat, 2005; Guzmán \& Sánchez, 2006).

Por último, en lo que respecta a las limitaciones del estudio, se destaca que la cantidad de participantes de la muestra seleccionada fue pequeña y que no se pueden plantear aspectos concluyentes y generalizables, así como también que la duración del programa de intervención fue relativamente corta. En este sentido, se sugiere como líneas de investigación futuras, llevar a cabo dicho proceso con una muestra más amplia, incrementando el número de sesiones, a fin de generar efectos más notorios en las dimensiones del pensamiento crítico que no mejoraron de manera significativa en los estudiantes.

Del mismo modo, se sugiere dar continuidad a la realización de programas de intervención de este tipo, considerando otros niveles de formación y carreras profesionales, que se encaminen al desarrollo de habilidades de pensamiento crítico de los universitarios, reflejándose así en la calidad educativa y profesional de las sociedades en el contexto latinoamericano.

\section{Referencias}

Acosta, C. (2002). Efectos del diálogo socrático sobre el pensamiento crítico en estudiantes universitarios. Psicología desde el Caribe, (10), 1-26.

Albornoz, J. (2017). Manual ASPADE: debate y argumentación para el desarrollo de pensamiento crítico. Santiago de Chile: ASPADE.

Amador, B. (2012). Percepciones sobre pensar críticamente en Colombia. Encuentros, (1), 6979.

Anganoy, A., Pantoja, C., Jurado, M., Vallejo, R. \& Botina, Z. (2017). Caracterización de las habilidades del pensamiento crítico y su relación con el desempeño académico (tesis de maestría), Universidad Pontificia Bolivariana, Putumayo, Colombia.

Arcila, M., Esteban, P., Gómez, J., Londoño, A., Londoño, N., López, S., Mercado, H., Montoya, N. \& Oviedo, M. (2017). Concurso de debate crítico. Conceptos operativos. Medellín: Editorial EAFIT.

Aznar, I. \& Laiton, I. (2017). Desarrollo de Habilidades Básicas de Pensamiento Crítico en el Contexto de la Enseñanza de la Física Universitaria. Formación universitaria, 10(1), 7178. Doi: $10.4067 /$ S0718-50062017000100008 
Bejarano, L., Galvan, F. \& López, B. (2013). Pensamiento crítico y motivación hacia el pensamiento crítico en estudiantes de psicología (tesis de maestría), Universidad de Manizales, Manizales, Colombia.

Beltrán, M. \& Torres, N. (2009). Caracterización de habilidades de pensamiento crítico en estudiantes de educación media a través del Test HCTAES. Zona Próxima, (11), 66-85.

Betancourth, S. (Septiembre de 2010). Desarrollo del pensamiento crítico a través de la controversia. Ponencia llevada a cabo en el Congreso Iberoamericano de Educación. Metas educativas 2021, Buenos Aires, Argentina.

Betancourth, S., Enríquez, A. \& Castillo, P. (2010). La controversia-socrática en el desarrollo del pensamiento crítico en estudiantes universitarios. Revista Virtual Universidad Católica del Norte, (29), 71-84.

Betancourth, S. (2015). Desarrollo del pensamiento crítico en docentes universitarios. Una mirada cualitativa. Revista Virtual Universidad Católica del Norte, (44), 238-252.

Betancourth, S., Muñoz, K. \& Rosas, T. (2017). Evaluación del pensamiento crítico en estudiantes de educación superior de la región de Atacama-Chile. Prospectiva. Revista de Trabajo Social e intervención social, (23), 199-223. Doi: https://doi.org/10.25100/prts.v0i23.4594

Bono, R. (2012). Diseños cuasi-experimentales y longitudinales. España: Universidad de Barcelona,

Buitrago, D., Higuita, A. \& Moreno, L. (2010). El desarrollo del pensamiento crítico a partir de las estrategias comunidad de indagación y aprendizaje basado en problemas (tesis de pregrado), Universidad de Antioquia, Medellín, Colombia.

Castro, M., Ruiz, J. \& Guzmán, F. (2018). Cruce de las pruebas nacionales Saber 11 y Saber Pro en Antioquia, Colombia: una aproximación desde la regresión geográficamente ponderada (GWR). Revista Colombiana de Educación, (74), 63-79. Doi: 10.17227/rce.num74-6898

Consejo Privado de Competitividad. (2013). Informe nacional de competitividad 2012-2013. Bogotá, Colombia: Puntoaparte.

Creamer, M. (2011). ¿Qué es y por qué pensamiento crítico? Curso de didáctica del pensamiento crítico, Ministerio de Educación de Ecuador. Recuperado de https://educacion.gob.ec/wp-content/uploads/downloads/2013/03/SiProfe-Didacticadel-pensamiento-critico.pdf

Creswell, J. (2014). Research Design: Qualitative, Quantitative, and Mixed Methods Approaches. California, EE. UU: Sage publications.

Dávila, M. (2018). Pensamiento crítico y rendimiento académico en los ingresantes del curso de estadística I en la Facultad de Ingeniería Económica, Estadística y Ciencias Sociales. Universidad Nacional de Ingeniería-2017 (tesis de maestría), Universidad Peruana Cayetano Heredia, Lima, Perú.

Difabio de Anglat, H. (2005). El critical thinking movement y la educación intelectual. Estudios sobre Educación, (9), 167-187. Doi: https://doi.org/10.15581/004.9.\%25p

Drago, C. (2017). Manual de apoyo docente: evaluación para el aprendizaje. Santiago de Chile: Editorial Universidad Central de Chile. 
Ennis, R., Millman, J. \& Tomko, T. (1985). Cornell Thinking Test Level X \& Level Z. Manual. California, EE. UU: Midwets Publications.

Flores, E., Miranda, M. \& Villasís, M. (2017). El protocolo de investigación VI: cómo elegir la prueba estadística adecuada. Estadística inferencial. Rev Alerg Mex, 64(3), 364-370. Doi: 10.29262/ram.v64i3.304

Freeley, A. \& Steinberg, D. (2009). Argumentation and Debate. Critical Thinking for Reasoned Decision Making. California, EE. UU: Wadsworth Cengage Learning.

Fuentes, C. \& Santibáñez, C. (2011): Diseñando debates: preliminares para un enfoque dialógico y crítico. En Cattani, A. Argomentare le proprie ragioni. Organizzare, condurre e valutare un dibattito. (pp. 111-133). Casoria, Italia: Loffredo Editore University Press.

Fuentes, C. (2011). Elementos para o desenho de um modelo de debate crítico na escola. En S. Leitão \& M. DaMianOvic (Orgs.). Argumentação na escola: o conhecimento em construção (pp. 115). Campinas, Brasil: Pontes.

García, L. \& Vázquez, A. (2018). Relación entre el pensamiento crítico y el desempeño académico en alumnos de escuela preparatoria. Educar, 54(2), 411-427. Doi: https://doi.org/10.5565/rev/educar.768

Godoy, M. \& Calero, K. (2018). Pensamiento crítico y tecnología en la educación universitaria. Una aproximación teórica. Revista espacios, 39(25), 36-41.

González, M. (2013). Enseñar a pensar: desafío ético de la educación crítica. Fundamentos, orientaciones y estrategias para el desarrollo del Pensamiento Crítico en estudiantes universitarios. Universidad Academia de Humanismo Cristiano, Chile. Recuperado de http://www.academia.cl/wp-content/uploads/2017/03/D9-DIDA-Manual-para-eldesarrollo-del-pensamiento-cri\%CC\%81 tico.pdf

Guzmán, S. \& Sánchez, P. (2006). Efectos de un programa de capacitación de profesores en el desarrollo de habilidades de pensamiento crítico en estudiantes universitarios en el Sureste de México. Revista Electrónica de Investigación Educativa, 8(2), 1-18.

Guzmán, S. \& Sánchez, P. (2008). Efectos del entrenamiento de profesores en el pensamiento crítico en estudiantes universitarios. Revista Latinoamericana de Estudios Educativos, 37(3-4), 189-199.

Hawes, G. (2003). Pensamiento crítico en la formación universitaria. Universidad de Talca: Instituto de Investigación y Desarrollo Educacional.

Hernández, R., Fernández, C. \& Baptista, L. (2006). Metodología de la Investigación. México: Mc. Graw Hill.

Laiton, I. (2010). Formación de pensamiento crítico en estudiantes de primeros semestres de educación superior. Revista Iberoamericana de Educación, 53(3), 1-7. Doi: https://doi.org/10.35362/rie5331730

Lander, E. (2001). Pensamiento crítico latinoamericano: la impugnación del eurocentrismo. Revista de Sociologia, (15), 13-25. Doi: 10.5354/0719-529X.2001.27766

Lara, J. \& Rodríguez, E. (2016). Fomento del desarrollo del pensamiento crítico en estudiantes de grado décimo desde situaciones cotidianas en la asignatura de Filosofía. Educación y Humanismo, 18(31), 343-357. Doi: http://dx.doi.org/10.17081/eduhum.18.31.1383

Leitão, S., De chiaro, S. \& Cano, M. (2016). El debate crítico. Un recurso de construcción del 
conocimiento en el aula. Textos. Didáctica de la Lengua y la Literatura, (73), 26-33.

Loaiza, Y. \& Osorio, L. (2018). El desarrollo de pensamiento crítico en ciencias naturales con estudiantes de básica secundaria en una Institución Educativa de Pereira - Risaralda. Diálogos sobre educación, 9(16), 1-24.

López, G. (2012). Pensamiento crítico en el aula. Docencia e Investigación, 1(22), 41-60.

Lozano, M. \& Cerrón, A. (2015). Pensamiento crítico y rendimiento académico de estudiantes de la Facultad de Educación de la Universidad Nacional del Centro del Perú. Horizonte de la Ciencia, 5(8), 105-110. Doi: https://doi.org/10.26490/uncp. horizonteciencia.2015.8.126

Manterola, C. \& Otzen, T. (2015). Estudios experimentales $2^{\mathrm{a}}$ parte. Estudios cuasiexperimentales. Int. J. Morphol, 33(1):382-387. Doi: http://dx.doi.org/10.4067/S071795022015000100060

Melo, L., Ramos, J. \& Hernández, P. (2017). La Educación Superior en Colombia: situación actual y análisis de eficiencia. Revista Desarrollo y Sociedad, (78), 59-111.

Minte, A. \& Ibagón, N. (2017). Pensamiento crítico: ¿competencia olvidada en la enseñanza de la historia?. Entramado, 13(2), 186-198. Doi: http://dx.doi.org/10.18041/entramado.2017v13n2.26228

Molina, C., Morales, G. \& Valenzuela, J. (2016). Competencia transversal pensamiento crítico: Su caracterización en estudiantes de una secundaria de México. Revista electrónica Educare, 20(1), 1-26. Doi: http://dx.doi.org/10.15359/ree.20-1.11

Monarca, H. (2013). Participación dialógica en la universidad: condición para el desarrollo del pensamiento crítico y el compromiso social. Revista Iberoamericana de Educación Superior, 4(9), 53-62. Doi: https://doi.org/10.1016/S2007-2872(13)71916-4

Montoya, J. \& Monsalve, J. (2008). Estrategias didácticas para fomentar el pensamiento crítico en el aula. Revista Virtual Universidad Católica del Norte, (25), 1-25.

Moreno, W. \& Velázquez, M. (2017). Estrategia didáctica para desarrollar el pensamiento crítico. REICE, 15(2), 53-73. Doi: https://doi.org/10.15366/reice2017.15.2.003

Mota, C. (2010). Desarrollo del pensamiento crítico a través del discurso argumentativo: una experiencia pedagógica en un curso de lectura y escritura. Entre Lenguas, (15), 11-23.

Núñez, S., Ávila, J. \& Olivares, S. (2017). El desarrollo del pensamiento crítico en estudiantes universitarios por medio del Aprendizaje Basado en Problemas. Revista iberoamericana de educación superior, 8(23), 84-103. Doi: 10.22201/iisue.20072872e.2017.23.3012

Olivares, S. (2016). Desarrollar el pensamiento crítico: decidiendo en que creer. En J. Valenzuela (Comp.). Competencias transversales para una sociedad basada en conocimiento. (pp. 170-188). Monterrey, México: Cengage Learning.

Ossa, C., Palma, M., Lagos, N. \& Díaz, C. (2018). Evaluación del pensamiento crítico y científico en estudiantes de pedagogía de una universidad chilena. Revista Electrónica Educare, 22(2), 1-18. Doi: http://dx.doi.org/10.15359/ree.22-2.12

Palacios, W., Álvarez, M., Moreira, J. \& Morán, C. (2017). Una mirada al pensamiento crítico en la Educación Superior. EDUMECENTRO, 9(4), 194-206.

Paul, R. \& Elder, L. (2003). La mini-guía para el pensamiento crítico. Conceptos y herramientas. 
Fundación para el Pensamiento Crítico. Recuperado de https://www.criticalthinking.org/resources/PDF/SP-ConceptsandTools.pdf

Paul, R. \& Elder, L. (2008). Critical thinking: strategies for improving student learning. Journal of Developmental Education, 32(1), 32-33.

Pinto, N. (2017). Sistematización de la experiencia El debate crítico como estrategia académica para fortalecimiento de las habilidades comunicativas y argumentativas en los estudiantes (tesis de pregrado), Corporación Universitaria Minuto de Dios, Bogotá, Colombia.

Prieto, J. (2008). Pensamiento crítico y universidad: Estrategias para la consolidación de una sociedad democrática en México. Investigación y ciencia, 1(42), 36-44.

Ramos, W. (2019). Argumentación, comunicación y falacias. Revista Góndola, Enseñanza y Aprendizaje de las Ciencias, 14(1), 162-164. Doi: https://doi.org/10.14483/23464712.14123

Rivas, S. \& Saiz, C. (2012). Validación y propiedades psicométricas de la prueba de pensamiento crítico PENCRISAL. Revista Electrónica de Metodología Aplicada, 17(1), 18-34.

Rivas, S. \& Saiz, C. (2016). Instrucción en pensamiento crítico: influencia de los materiales en la motivación y rendimiento. Revista Latinoamericana de Estudio Educativos, 12(1), 91106.

Rivas, S., Morales, P. \& Saiz, C. (2014). Propiedades psicométricas de la adaptación peruana de la prueba de pensamiento crítico PENCRISAL. Avaliação Psicológica, 13(2), 257-268.

Robles, S., Cisneros, L. \& Guzmán, C. (2016). Evaluación del nivel de pensamiento crítico en estudiantes universitarios de pregrado y posgrado. El caso de un Centro Universitario Temático de la Universidad de Guadalajara. Revista de Educación y Desarrollo, (39), 19.

Roca, J. (2013). El desarrollo del pensamiento crítico a través de diferentes metodologías docentes en el grado de enfermería (tesis de doctorado), Universidad Autónoma de Barcelona, Barcelona, España.

Saiz, C. \& Rivas, S. (2008). Evaluación en pensamiento crítico: una propuesta para diferenciar formas de pensar. Revista ERHO, (22), 25-66.

San Martín, P. (2011). Aplicación de la estadística no paramétrica en el área de rehabilitación. Rehabil. Integral, 6(2), 93-99.

Santiago, J. (2016). La acción didáctica de las Ciencias Sociales y el desarrollo del pensamiento crítico. Educación y Humanismo, 18(31), 241-256. Doi: http://dx.doi.org/10.17081/eduhum.18.31.1377

Steffens, E., Ojeda, D., Martínez, J., Hernández, H. \& Moronta, Y. (2018). Presencia del pensamiento crítico en estudiantes de educación superior de la Costa Caribe Colombiana. Revista Espacio, 39(3), 1-14.

Vásquez, B., Pleguezuelos, C. \& Mora, M. (2017). Debate como metodología activa: una experiencia en Educación Superior. Universidad y Sociedad, 9(2), 134-139.

Vélez, C. (2013). Una reflexión interdisciplinar sobre el pensamiento crítico. Revista Latinoamericana de Estudios Educativos, 9(2), 11-39. 
Willson, J. (2012). Thinking critically about critical thinking. Educational Philosophy \& Theory, 44(5), 464-479. Doi: 10.1111/j.1469-5812.2010.00673 